\title{
CICLICIDAD DE LAS TRANSFERENCIAS SOCIALES EN COSTA RICA
}

\author{
Alejandro Abarca ${ }^{1}$ \\ Juan Robalino ${ }^{2}$ \\ Luis Hall ${ }^{3}$ \\ Esteban Pérez ${ }^{4}$
}

Recibido: $13 / 07 / 2018$

Aprobado: 09/10/2018

\section{RESUMEN}

\begin{abstract}
En este artículo, se busca determinar si las transferencias sociales del Gobierno en Costa Rica han sido procíclicas o contra-cíclicas. Para examinar la presencia de ciclicidad, se utilizó correlaciones entre las series de producción y de transferencias, luego de que las variables fueran filtradas con el método de Hodrick-Prescott. También se utilizan métodos de regresión explicando el crecimiento de las transferencias con el crecimiento de la producción, tanto de forma contemporánea como rezagada. Se utilizaron datos trimestrales para las estimaciones y se encontró que las transferencias contemporáneas no responden con fuerza al ciclo; sin embargo, sí responden al rezago (trimestre anterior), donde hay una fuerte relación contra-cíclica. Por otra parte, se determina que la mayoría de los programas sociales importantes tienen una relación negativa con la producción trimestral rezagada. Esto implica que, cuando la producción se desacelera, hay un aumento en el siguiente trimestre del gasto en los programas sociales. Concluimos que, en Costa Rica, los programas sociales, por su ciclicidad, han tenido el potencial de reducir los impactos negativos de los choques económicos sobre las poblaciones más vulnerables.
\end{abstract}

PALABRAS CLAVE: CICLOS ECONÓMICOS, GASTO PÚBLICO, GOBIERNO, FLUCTUACIONES ECONÓMICAS, PRODUCTO INTERNO BRUTO, TRANSFERENCIAS SOCIALES.

CLASIFICACIÓN JEL: H50, E320, I380

\section{ABSTRACT}

In this article, we try to determine if the social transfers of the Government in Costa Rica have been pro-cyclical or counter-cyclical. To examine the presence of cyclicity, correlations were used between the production and transfer series, after the variables

1 Universidad de Costa Rica, Dirección de Asignaciones Familiares de Costa Rica; Código Postal 10101; San José, Costa Rica, alejoag2@gmail.com

2 Universidad de Costa Rica, Escuela de Economía; Código Postal 11501-2060, San José, Costa Rica; robalino@catie.ac.cr

3 Universidad de Costa Rica, Escuela de Economía; Código Postal 11501-2060, San José, Costa Rica; ljh223@nyu.edu

4 Universidad de Costa Rica, Escuela de Economía; Código Postal 11501-2060, San José, Costa Rica; eperezb96@gmail.com 
were filtered using the Hodrick-Prescott method. Regression methods are also used to explain the growth of transfers with the growth of production, both contemporaneously and lagging behind. Quarterly data were used for the estimates and it was found that the contemporary transfers do not respond strongly to the cycle; however, they do respond to the lag (previous quarter), where there is a strong counter-cyclical relationship. On the other hand, it is determined that most of the important social programs have a negative relationship with the lagged quarterly production. This implies that, when production slows down, there is an increase in spending on social programs in the following quarter. We conclude that, in Costa Rica, social programs, due to their cyclicality, have had the potential to reduce the negative impacts of economic shocks on the most vulnerable populations.

KEYWORDS: GOVERNMENT, ECONOMIC FLUCTUATIONS, GROSS DOMESTIC PRODUCT, SOCIAL TRANSFERS, ECONOMIC CYCLE, PUBLIC EXPENDITURE

JEL CLASIFICATION: H50, E320, I380

\section{INTRODUCCIÓN}

La ciclicidad del gasto del Gobierno ha sido un tema ampliamente estudiado en los países desarrollados (Abbott \& Jones, 2012a, 2012b, 2014, 2016; Furceri, 2010; Lane, 2003). Solo ha sido hasta recientemente, que la literatura ha empezado a enfocarse en países en vías de desarrollo (Ahuja \& Murthy, 2017; Bello \& Ruiz, 2009; Prasad \& Gerecke, 2010). Una parte importante del gasto del Gobierno en estos países son las transferencias sociales. Este tipo de gasto es un propulsor importante del consumo de los hogares, especialmente para aquellos más vulnerables. Por lo tanto, entender si existe ciclicidad en transferencias del Gobierno es clave para determinar si este rubro del gasto ha acentuado o diezmado la magnitud de los ciclos económicos. La volatilidad del producto y los niveles de pobreza hacen que sea primordial utilizar todas las herramientas al alcance del Estado para reducir los efectos adversos de los ciclos económicos en estos países.

Además de tener propósitos de política pública, las transferencias fiscales juegan un rol importante dentro de la ciclicidad de una economía. En el caso de los países que forman parte de la Organización para la Cooperación y el Desarrollo Económicos (OCDE), se ha encontrado que, para que haya ajustes fiscales efectivos en el largo plazo, se deben llevar a cabo cambios en las transferencias y los salarios del sector público (Alesina \& Perotti, 1995). Este resultado es, además, importante, al considerar que existen indicios y teoría que señala que no hay evidencia de que el gasto de Gobierno sea procíclico y los impuestos contracíclicos para EE. UU. (Barseghyan, Battaglini, \& Coate, 2013), y que aumentos en el gasto de Gobierno tienen un efecto multiplicador positivo en las economías de la OCDE (Fatás \& Mihov, 2001a). Asimismo, se ha establecido una fuerte correlación negativa entre el tamaño de Gobierno y la volatilidad del PIB en los países de la OCDE (Fatás \& Mihov, 2001b). Toda esta evidencia no solo sugiere que las transferencias son elementos determinantes de la ciclicidad de una economía, sino también que pueden tener efectos de corto y largo plazo.

En países desarrollados, los gastos sociales son contra-cíclicos (Abbott \& Jones, 2012a). Sin embargo, hay evidencia de que esta contra-ciclicidad está limitada por la capacidad de endeudamiento del Gobierno (Abbott \& Jones, 2012b). Son los países desarrollados los que muestran más ciclicidad en los gastos de seguridad social (Arze del Granado, Gupta, \& Hajdenberg, 2013; Prasad \& Gerecke, 2010). En países asiáticos, los gastos en seguridad social muestran patrones procíclicos (Ahuja \& Murthy, 2017). En Latinoamérica, los resultados son similares. Existe evidencia de que choques financieros tienen efectos negativos sobre el gasto en programas sociales (Gautam, 2013). Evidencia de pro-ciclicidad en el gasto social también se ha encontrado para Argentina, Colombia, 
México, Panamá, Perú, República Dominicana, Uruguay y Venezuela (Bello \& Ruiz, 2009). Estos resultados implican que, en nuestra región, las transferencias no contribuyen a la reducción de los efectos adversos de las recesiones.

En esta línea, el objetivo de este documento es determinar si las transferencias del Gobierno en Costa Rica han sido procíclicas o contra-cíclicas. El estudio se centra en las transferencias del sector social, donde se incluyen las transferencias directas a los hogares en desventaja, a través de los programas sociales.

Para examinar la hipótesis de la presencia de ciclicidad se utilizan dos métodos. Primero, se analizan las correlaciones entre las series de producción y de transferencias, luego de que las variables hayan sido filtradas con el método Hodrick-Prescott. Luego, se utilizan regresiones para explicar el crecimiento de las transferencias con el crecimiento de la producción, tanto contemporáneo como rezagado.

Una contribución clave en este documento es la posibilidad de analizar la ciclicidad del gasto del Gobierno desagregado en forma trimestral. Esto es ventajoso, ya que la agregación anual no solamente reduce el número de observaciones, sino que también elimina variabilidad en las series del producto, lo que no permite observar algunos ciclos. Se determina que las transferencias contemporáneas no responden con fuerza al ciclo; sin embargo, sí responden al rezago (trimestre anterior) donde hay una fuerte relación contra-cíclica. La mayoría de los programas sociales importantes tienen una relación negativa con la producción rezagada. Esto implica que cuando la producción se desacelera hay un aumento en el siguiente trimestre del gasto en los programas sociales.

Para el caso de Costa Rica, concluimos que los programas sociales han permitido reducir los impactos negativos de los choques económicos sobre las poblaciones más vulnerables. Además, recalcamos la importancia de hacer este tipo de análisis a un nivel de desagregación temporal que permita capturar en forma adecuada las fluctuaciones que desaparecen al agregar los datos en forma temporal.

El documento está organizado de la siguiente manera. En la siguiente sección, se describen los antecedentes que incluye una discusión sobre la evolución de la producción y de los programas sociales. En la tercera sección, se describen los datos. La metodología se discute en la cuarta sección, mientras que los resultados se muestran en la quinta sección. Finalmente, se presentan las conclusiones en la última sección.

\section{ANTECEDENTES}

\subsection{Evolución del producto interno bruto en Costa Rica}

A partir de 1991, el producto interno bruto real de Costa Rica ha presentado un crecimiento anual promedio de 4,4\%, aproximadamente (ver gráfico 1). Es importante destacar el período de 1995-1996, cuando la producción creció a una tasa de 1,22\%, baja en comparación con los años previos y con la tasa de crecimiento promedio. Esto fue debido a que, en primer lugar, la política fiscal generó problemas con la deuda pública interna del país; en segundo lugar, la caída del poder adquisitivo de los salarios y; en último lugar, la presencia del huracán César, el cual generó efectos negativos en las actividades económicas, principalmente en la costa pacífica (Ramírez, 1997).

En el gráfico 1 también se puede observar una tasa de crecimiento alta en 1992, debido a la entrada importante de capitales privados de corto plazo, sin un aumento correspondiente del ahorro interno o de la formación de capital (Sauma F., Sánchez Cantillo, Chacón, \& Sáenz, 2010). También se observa un aumento en 1998, debido al incremento de la inversión extranjera directa (CEPAL, 1999) y, en el 2007, debido al incremento de la inversión bruta fija y las exportaciones (CEPAL, 
2008). En el período de 2009, la tasa de crecimiento del PIB cayó hasta llegar a ser casi un -1 \%, debido al efecto de la crisis mundial del 2008. Por otra parte, en los demás períodos, la tasa de crecimiento mantuvo niveles cercanos al promedio.

\section{GRÁFICO 1}

EVOLUCIÓN DEL PIB REAL Y TASA DE CRECIMIENTO ANUAL EN COSTA RICA
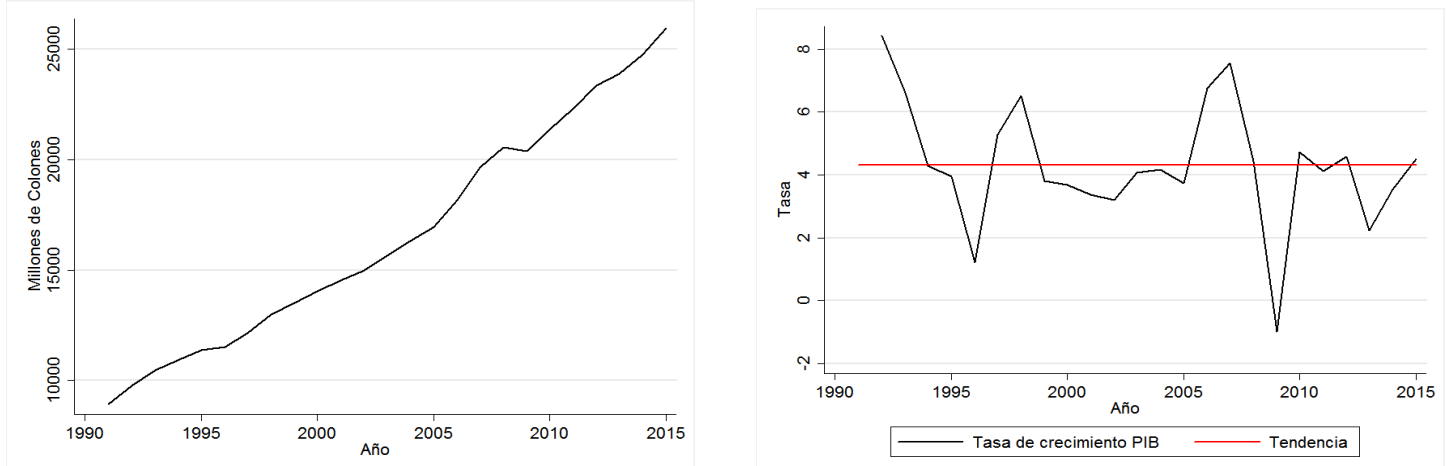

Fuente: Elaboración propia con datos del Banco Central de Costa Rica

En el gráfico 2, se observa el comportamiento del PIB y su tasa de crecimiento trimestral. Se puede observar una tendencia congruente con los gráficos del PIB anual; sin embargo, se denota la existencia de ciclos en el producto. Se observa que, en los segundos trimestres de cada año, el PIB disminuye, mientras que, en el primer semestre del año, disminuye ocasionalmente. Además, en el tercer $y$ en el cuarto semestre del año, se nota un incremento.

\section{GRÁFICO 2}

EVOLUCIÓN DEL PIB REAL TRIMESTRAL EN COSTA RICA

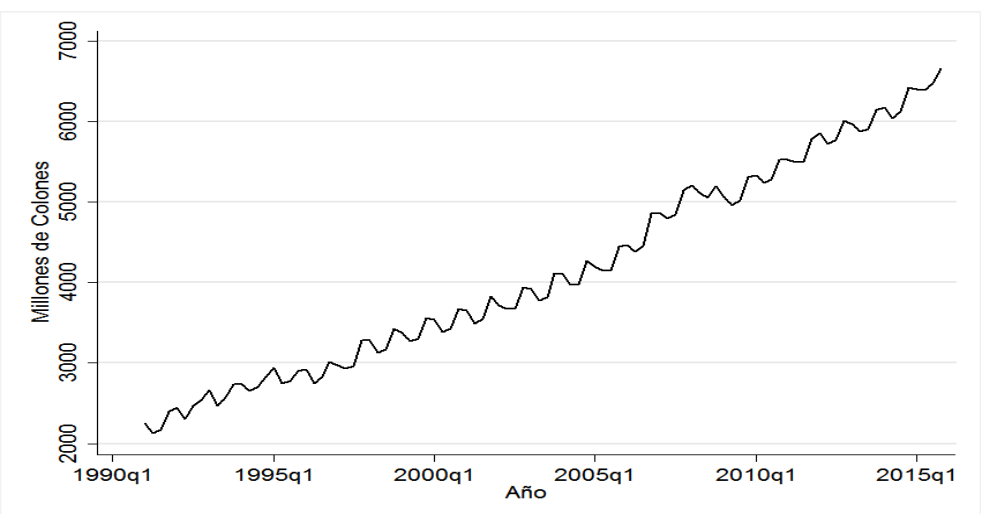

Fuente: Elaboración propia con datos del Banco Central de Costa Rica

\subsection{Transferencias sociales}

En este documento, consideramos las transferencias de programas sociales del Gobierno. A continuación, se describe el funcionamiento de las transferencias sociales del Estado hacia la población. 
Las transferencias de programas sociales para Costa Rica son, en su gran mayoría, manejadas por el Fondo de Desarrollo Social y Asignaciones Familiares (FODESAF), el cual es la principal herramienta de política social del país. Este fondo fue creado en 1974, con el fin de financiar los programas del sector social $y$, en especial, los programas sociales destinados a reducir la pobreza ${ }^{5}$. El fondo actualmente representa el mayor instrumento de financiamiento de la política social selectiva del país y sus recursos se usan para atender a cerca de un millón de beneficiarios en varios programas sociales, por medio de subsidios para la vivienda, pensiones no contributivas, becas estudiantiles, comedores estudiantiles, red de cuido infantil, entre otros (FODESAF, 2018).

Dicho fondo es administrado por la Dirección General de Desarrollo Social y Asignaciones Familiares (DESAF) que, a su vez, es una dependencia técnica, independiente del Ministerio de Trabajo y Seguridad Social. Dicha dirección tiene como responsabilidad la administración de los recursos del fondo, así como supervisar y controlar la ejecución de estos por parte de las instituciones y programas sociales que los reciben

El FODESAF es, entonces, un fondo permanente, el cual es financiado por recursos nacional y cuenta con aportes del sector público y privado. Actualmente, cuenta con dos fuentes de financiamiento: por un lado, el Ministerio de Hacienda incluye cada año, en el presupuesto ordinario anual de la República, una asignación equivalente a 593.000 salarios base utilizados por el Poder Judicial para fijar multas y penas por la comisión de diferentes infracciones, proveniente de la recaudación del impuesto sobre las ventas; por otro lado, los patronos públicos y privados aportan al fondo un 5\% sobre el total de sueldos y salarios que paguen mensualmente a sus trabajadores. En el gráfico 3 se muestra cómo ha evolucionado el presupuesto del FODESAF entre el 2005 y el 2015 y su tamaño con respecto al PIB de Costa Rica.

GRÁFICO 3

PRESUPUESTO DE FODESAF EN MILLONES DE DÓLARES Y SU PORCENTAJE CON RESPECTO AL PIB

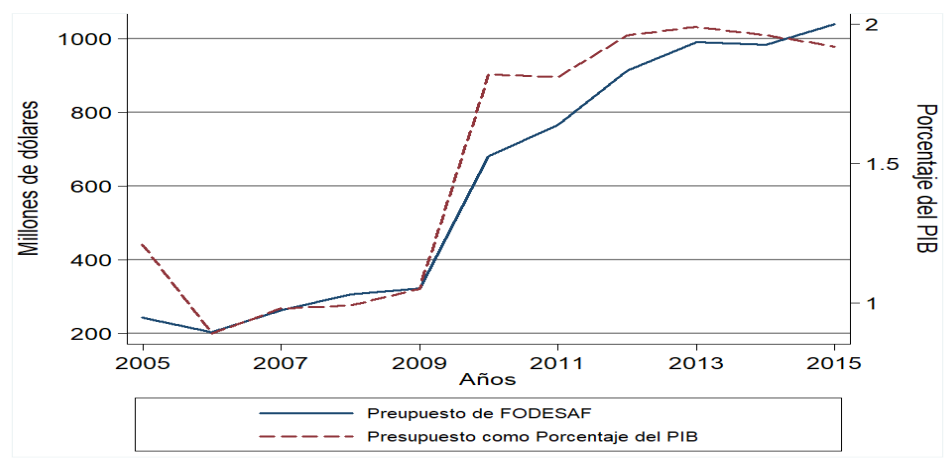

Fuente: Elaboración propia con datos del Banco Central de Costa Rica

Destaca, por una parte, como el tamaño del FODESAF tiene importantes implicaciones macroeconómicas al ser alrededor del 2\% del PIB de Costa Rica. También se puede observar cómo, a partir del 2010, el presupuesto del fondo tuvo un aumento muy significativo con respecto a años anteriores. Esto coincide con la reforma de la Ley 5662 -la cual dio origen al fondo y establece las

Este fondo fue creado bajo la ley 5662. Ley de Desarrollo Social y Asignaciones Familiares, N 5662. 
dos fuentes de financiamiento mencionadas previamente en este texto- mediante la Ley 8787. Previo a esta reforma, los ingresos del FODESAF correspondían a un $20 \%$ de los ingresos recolectados por impuesto de ventas y al 5\% del total de sueldos y salarios del país (Art. 26, Ley $\mathrm{N}^{\circ} 8783$ ). Además de mayores ingresos para el fondo, esta reforma dio a DESAF más responsabilidades y mayores poderes de regulación y supervisión de los recursos del FODESAF.

Cabe destacar que los recursos FODESAF solo deben ser utilizados para financiar los productos o servicios provistos por cada institución. Es decir, no pueden financiar gastos administrativos de las instituciones que reciben estos recursos, salvo algunas excepciones específicas de ley. De tal manera que los egresos del FODESAF corresponden, en su gran mayoría, a los recursos destinados efectivamente a los beneficiarios $y$ no a gastos operativos por parte de las instituciones ejecutoras de los programas sociales.

En cuanto a la distribución de los recursos del fondo, alrededor del 95\% de estos tienen destinos específicos asignados por leyes o convenios. Por ejemplo, por ley, al menos un 2,6 \% de los recursos de FODESAF deben ser destinados a programas de nutrición infantil; así mismo, el Instituto Nacional de las Mujeres (INAMU) debe recibir un $2 \%$; los comedores escolares del Ministerio de Educación Pública (MEP), al menos un 5,18 \%; el Patronato Nacional de la Infancia (PANI), un mínimo de 2,59 \%; el Instituto Mixto de Ayuda Social (IMAS), al menos un 4 \%; los subsidios a la vivienda, un $18,3 \%$ de los recursos del fondo; y las pensiones no contributivas, un 10,35 \%. Además de estos porcentajes específicos -que suelen ser montos mínimos-, existen otros programas cuyo financiamiento proviene del FODESAF por decreto ejecutivo o convenio. De tal manera que la distribución del gasto social selectivo del país tiene poco lugar para la discrecionalidad. Más específicamente, en la ley del FODESAF, poco más del 50\% de su presupuesto ya está definido por ley (Art. 26, Ley $\mathrm{N}^{\circ} 8783$ ).

Para contrastar cómo se aplica esta ley en la práctica, en el cuadro 1 se muestra la distribución del gasto del FODESAF para el 2016 por tipo de programas sociales. Del cuadro se desprende que muchos de los programas sociales reciben más dinero del que la ley del FODESAF indica. Por ejemplo, los "Programas de bienestar social (varios)" son ejecutados por el IMAS, el cual por ley debería recibir al menos un 4\% del presupuesto total del FODESAF, pero en la práctica recibe más de cinco veces lo establecido en la ley. Similarmente, los comedores escolares reciben más de cuatro veces el mínimo exigido por ley.

Hay que señalar, sin embargo, que estos giros de recursos por encima de lo establecido por ley no van en detrimento necesariamente del financiamiento de estos programas. Este hecho indica que para que estos se desempeñen de manera apropiada, requieren de más recursos que los estipulados por ley. No obstante, esto refuerza la idea de que el gasto social selectivo del país tiene poco lugar para la discrecionalidad ${ }^{6}$. 
CUADRO 1

COMPOSICIÓN DEL GASTO DEL FODESAF POR TIPO DE PROGRAMA, 2016

\begin{tabular}{lr}
\hline \multicolumn{1}{c}{ Country } & 2011:QIV \\
\cline { 2 - 2 } & \multicolumn{1}{c}{ own r } \\
\hline Pensiones no contributivas y seguro social provisto por el estado & 30,00 \\
Programas de empleo & 3,12 \\
Programas para personas discapacitadas & 0,87 \\
Programas para el cuido de adultos mayores & 2,24 \\
Subsidios de vivienda & 18,26 \\
Becas estudiantiles & 3,35 \\
Programas de alimentación y cuido infantil & 5,12 \\
Programas de protección infantil & 2,85 \\
Programas de bienestar social (varios) & 21,33 \\
Comedores escolares & 8,70 \\
Otros programas & 4,17 \\
\hline
\end{tabular}

Fuente: FODESAF (2016)

En este estudio, se priorizará el análisis de pensiones no contributivas (30\%), subsidios de vivienda $(18,26 \%)$, becas estudiantiles $(3,35 \%)$, programas de alimentación y cuido infantil $(5,12$ $\%)$, programas de bienestar social a través del IMAS (21,33 \%) y comedores escolares $(8,70 \%)$.

\section{METODOLOGÍA}

\subsection{Datos}

En este trabajo se utilizaron datos trimestrales. El período de los datos va desde el primer trimestre de 1991 hasta el último de 2015. A continuación, se detalla la disponibilidad de datos.

Los datos trimestrales provienen de dos fuentes principales: las bases de datos del Banco Central de Costa Rica (BCCR) y los informes de ejecución de programas sociales de la DESAF (ver cuadro 3$)$.

Los datos trimestrales del PIB están expresados en términos constantes de junio del 2012. Asimismo, con el deflactor del PIB trimestral se expresa en términos reales los valores de las transferencias sociales. En el siguiente cuadro se detalla la disponibilidad de datos para los programas sociales considerados. 
CUADRO 2

DISPONIBILIDAD DE DATOS TRIMESTRALES POR PROGRAMA SOCIAL

\begin{tabular}{lr}
\hline \multicolumn{1}{c}{ Programas Sociales } & Total de transferencias \\
\hline Subsidios de Vivienda (Bonos) & $1988-2015$ \\
Pensiones No Contributivas (PNC) & $2000-2015$ \\
Asegurados por cuenta del Estado & ND \\
Fondo Nacional de Becas (FONABE) & $2000-2015$ \\
Instituto Mixto de Ayuda Social (IMAS) & $2000-2015$ \\
Comedores Escolares (PANEA) & $2000-2015$ \\
Programas de cuido y nutrición infantil (CENCINAI) & $2000-2015$ \\
Avancemos & $2010-2015$ \\
\hline
\end{tabular}

Fuente: Elaboración propia con datos de DESAF y Banco Central de Costa Rica

\subsection{Estrategia empírica}

Con el fin de determinar el comportamiento existente entre las transferencias del Gobierno y el producto interno bruto en Costa Rica, se aplican dos métodos correlaciones y regresiones. Estos permitirán comprender de una manera formal la relación entre estas variables. El principal interés es identificar el comportamiento de las transferencias que realiza el Gobierno durante el ciclo económico y, en particular, poder fundamentar si existe o no una relación de co-movimiento. Si hay co-movimiento, significa que las transferencias responden en la misma dirección al desempeño económico agregado del producto interno bruto, lo que significa que las variables son pro-cíclicas. Si en su defecto, las variables no responden o responden en la dirección contraria al movimiento del PIB, las variables son acíclicas o contra-cíclicas, respectivamente.

\section{Correlaciones entre ciclos de las variables}

Un primer esquema analítico ampliamente empleado en la literatura macroeconómica de ciclos económicos busca identificar el componente cíclico del PIB y de las series macroeconómicas de interés $y$, posteriormente, valorar su correlación. En este esquema, formalmente no se puede señalar una causalidad entre las series, pero si reforzar el estudio de los hechos estilizados de interés que permitan afirmar si existe o no correlación entre las variables de interés.

Con el fin de establecer dicha relación, en primera instancia, se procede a filtrar con el método Hodrik-Prescott las series de tiempo de las transferencias del Gobierno y del producto interno. El objetivo es centrarse en el componente cíclico, por lo tanto, el filtro elimina el componente de baja frecuencia de la serie, es decir su componente de tendencia o crecimiento. Para este filtro, se utilizó un lambda de 1600 para los datos trimestrales.

A saber, sea $y_{t}$ la serie de tiempo de interés en el momentot, es decir, algún tipo de transferencia o el producto interno bruto en términos reales. Si se representa el término de tendencia y el cíclico respectivamente por $Y_{\text {tendencia,t }}$ y $Y_{\text {ciclo,t }}$ entonces el componente cíclico es:

$$
Y_{\text {ciclo,t }}=Y_{\mathrm{t}}-Y_{\text {tendencia,t }}
$$


En la próxima sección, una vez filtrada cada una de las series de transferencias y del producto interno bruto, se calculará su correlación en su componente cíclico. Las correlaciones positivas indican co-movimiento en la misma dirección, por lo que la expansión económica conlleva un movimiento paralelo, no necesariamente causal, de las transferencias de Gobierno. Por su parte, las correlaciones negativas indican que la expansión económica más bien lleva a una contracción de las transferencias. Este análisis de correlación presenta la restricción de estar potencialmente afectado por la diferencia en la volatilidad de las muestras de las series empleadas (Forbes, \& Rigobon, 2002; Lane, 2003). Este es potencialmente el caso en estudios que incluye distintos países donde este problema puede estar más latente y menos en un estudio de un solo país como el presente caso.

\subsubsection{Métodos de regresión}

Una vez establecidos los niveles de correlación para el caso de Costa Rica, se aplican métodos de regresión lineal para asociar el crecimiento del producto interno bruto del periodo contemporáneo y rezagado con el crecimiento del gasto en transferencias. Para ello, empleando la serie descrita en la sección anterior, $y$ tomando su tasa de crecimiento medida por las diferencias logarítmicas de la serie, se aplica la siguiente regresión:

$$
\log \left(G_{t}\right)-\log \left(G_{t-1}\right)=\alpha+\beta t\left(\log \left(P I B_{t}\right)-\log \left(P I B_{t-1}\right)\right)+\beta_{t-1}\left(\log \left(P I B_{t-1}\right)-\log \left(P I B_{t-2}\right)\right)+\varepsilon t
$$

En este caso, los coeficientes $\beta_{t} y \beta_{(t-1)}$ son la medida de relación entre el crecimiento del gasto en transferencias con el crecimiento contemporáneo y rezagado del PIB respectivamente. Como sugieren otros autores, este coeficiente mide el grado de elasticidad del gasto público en transferencias con respecto al crecimiento del producto (Arreaza, Sorensen, \& Yosha, 1998; Lane, 2003; Sorensen, Wu, \& Yosha, 2001). Un valor positivo de dicho coeficiente indica un comportamiento procíclico. Un valor por encima de la unidad reporta una reacción más que proporcional de las transferencias. Esto significa que las transferencias incrementan a una tasa mayor al incremento porcentual que sufrió el producto interno bruto. Un valor negativo de dicho coeficiente indica un comportamiento contra-cíclico. Un valor por debajo de menos uno refleja una reacción más que proporcional de las transferencias. Esto significa que las transferencias decrecen a una tasa mayor al incremento porcentual que sufrió el Producto Interno Bruto.

\section{RESULTADOS}

En esta sección se aplican los mismos métodos descritos en la sección de metodología para las transferencias sociales con datos trimestrales.

\subsubsection{Correlaciones utilizando el filtro HP}

En los cuadros 3 y 4, se ha realizado el análisis enfocándose en las transferencias sociales. Se obtiene evidencia estadística que nos permite rechazar la ausencia de una relación entre los programas de transferencias sociales y el producto. Se encuentran relaciones negativas entre el PIB trimestral contemporáneo y los programas de PANEA y Avancemos. Sin embargo, el programa de atención y salud CENCINAI es procíclico al producto contemporáneo. Además, se observan co-movimientos entre el PIB trimestral rezagado y varios programas de las transferencias sociales, tales como RNC, IMAS, PENEA y CENCINAI. 
CUADRO 3

CORRELACIÓN ENTRE LAS TRANSFERENCIAS SOCIALES Y EL PRODUCTO INTERNO

BRUTO EN TÉRMINOS CONSTANTES

VARIADAS FILTRADAS CON EL MÉTODO HP

\begin{tabular}{lrrr}
\hline \multirow{2}{*}{ Country } & \multicolumn{1}{c}{ Trimestral } & Trimestral & \multicolumn{1}{c}{ Trimestral } \\
\cline { 2 - 4 } & Contemporáneo & Rezagado+ & Rezagado++ \\
\hline Austria & 3,30 & 72,3 & 0,92 \\
Bonos & $-0,074$ & $-0,174$ & $-0,084$ \\
RNC & $-0,136$ & $-0,225^{*}$ & $-0,195$ \\
FONABE & $-0,130$ & 0,010 & 0,028 \\
IMAS & 0,064 & $-0,320^{* *}$ & 0,005 \\
PANEA & $-0,295^{* *}$ & $-0,233^{*}$ & $-0,288^{* *}$ \\
CENCINAI & $0,232^{*}$ & $-0,493^{* * *}$ & $0,233^{*}$ \\
Avancemos & $-0,612^{* *}$ & $-0,157$ & $-0,606^{* *}$ \\
\hline
\end{tabular}

+ Con respecto al trimestre anterior

++ Con respecto al mismo trimestre del año anterior.

Nota: se usó un lambda de 1600 para el filtro de Hoddrick y Prescott en los datos trimestrales.

\subsubsection{Regresiones}

Al emplear datos trimestrales, se puede rechazar dicho comportamiento acíclico a favor de respuestas contra-cíclicas y pro-cíclicas de algunos componentes. En particular, la mayor parte de los componentes del gasto en transferencias sociales muestra un comportamiento acíclico en relación con el producto contemporáneo. Solamente cuando se analiza la relación entre el crecimiento rezagado del producto y las transferencias es que se encuentran varios coeficientes negativos, ya sea medido con respecto al trimestre anterior o con respecto al mismo trimestre del año anterior. Esto ocurre en varios programas sociales grandes, como es el caso de RNC, IMAS, PANEA, $y$ CENCINAI. 


\section{CUADRO 4 \\ COEFICIENTES DE REGRESIÓN ENTRE CRECIMIENTO DEL PIB Y CRECIMIENTO DE LAS TRANSFERENCIAS DEL GOBIERNO}

\begin{tabular}{|c|c|c|c|c|c|}
\hline \multirow[t]{2}{*}{ Modelo } & 3 & \multicolumn{2}{|c|}{4} & \multicolumn{2}{|c|}{5} \\
\hline & $\begin{array}{l}\text { Crecimiento } \\
\text { con respecto } \\
\text { al mismo } \\
\text { trimestre del } \\
\text { año anterior }\end{array}$ & \multicolumn{2}{|c|}{$\begin{array}{l}\text { Crecimientos con } \\
\text { respecto al mismo } \\
\text { trimestre del año } \\
\text { anterior }\end{array}$} & \multicolumn{2}{|c|}{$\begin{array}{l}\text { Crecimiento con } \\
\text { respecto al trimestre } \\
\text { anterior }\end{array}$} \\
\hline \multirow[t]{2}{*}{ Datos } & Trimestrales & \multicolumn{2}{|c|}{ Trimestrales } & \multicolumn{2}{|c|}{ Trimestrales } \\
\hline & Contemp. & Contemp. & Rezago & Contemp. & Rezago \\
\hline \multirow[t]{2}{*}{ Bonos } & $\begin{array}{r}\text { trimestre del } \\
\text { año }\end{array}$ & $5,319^{* *}$ & $-5,160 * *$ & 0,564 & $-1,472$ \\
\hline & anterior & $\begin{array}{r}\text { Crecimiento } \\
\text { con }\end{array}$ & $(2,411)$ & $(1,253)$ & $(1,234)$ \\
\hline \multirow[t]{2}{*}{$\mathrm{RNC}$} & $\begin{array}{r}\text { respecto al } \\
\text { trimestre }\end{array}$ & 1,182 & $-1,523$ & 0,639 & $-2,928 * *$ \\
\hline & anterior & $(3,331)$ & $(3,328)$ & $(1,436)$ & $(1,438)$ \\
\hline \multirow[t]{2}{*}{ FONABE } & 0,629 & 14,87 & $-17,54$ & $-21,84$ & 14,29 \\
\hline & $(16,38)$ & $(33,95)$ & $(33,92)$ & $(14,78)$ & $(14,80)$ \\
\hline \multirow[t]{2}{*}{ IMAS } & 2,287 & 7,337 & $-6,344$ & 2,782 & $-14,61^{* * *}$ \\
\hline & $(3,179)$ & $(6,839)$ & $(6,833)$ & $(2,392)$ & $(2,395)$ \\
\hline \multirow[t]{2}{*}{ PANEA } & $-19,46$ & $-130,3$ & $147,0^{*}$ & $-51,08$ & $-107,6^{* * *}$ \\
\hline & $(52,64)$ & $(80,65)$ & $(80,58)$ & $(33,83)$ & $(33,87)$ \\
\hline \multirow[t]{2}{*}{ CENCINAI } & 17,73 & $-43,55$ & 50,98 & $-3,542$ & $-39,81^{* *}$ \\
\hline & $(22,36)$ & $(35,31)$ & $(35,28)$ & $(15,19)$ & $(15,21)$ \\
\hline \multirow[t]{2}{*}{ AVANCEMOS } & 9,719 & $-149,0$ & 177,6 & $-77,82$ & $-78,18$ \\
\hline & $(154,2)$ & $(162,6)$ & $(157,4)$ & $(70,17)$ & $(68,89)$ \\
\hline
\end{tabular}

Errores estándar en paréntesis. ${ }^{* * *} p<0.01,{ }^{* *} p<0.05,{ }^{*} p<0.1$. Nota: se usó un lambda de 1600 para el filtro de Hoddrick y Prescott en los datos. 


\section{CONCLUSIONES}

El objetivo de este documento fue determinar si las transferencias del Gobierno en Costa Rica han sido procíclicas o contra-cíclicas. El estudio se enfocó en las transferencias del sector social, en las cuales se incluyen aquellas transferencias directas a los hogares en desventaja a través de los programas sociales.

Para examinar la hipótesis de la presencia de ciclicidad, se utilizó correlaciones entre las series de producción y de transferencias luego de que las variables fueron filtradas con el método Hodrick-Prescott. También se utilizaron regresiones explicando el crecimiento de las transferencias con el crecimiento de la producción, tanto contemporáneo como rezagado.

Por medio de los métodos de análisis, se logra identificar patrones de ciclicidad claros. Se encuentra que las transferencias contemporáneas no responden con fuerza al ciclo; sin embargo, sí responden al rezago (trimestre anterior), donde hay una fuerte relación contra-cíclica. La mayoría de los programas sociales importantes tienen una relación negativa con la producción rezagada. Esto implica que cuando la producción se desacelera, existe un aumento en el siguiente trimestre del gasto en los programas sociales.

Para el caso de Costa Rica, se concluye que los programas sociales, por su ciclicidad, han tenido el potencial de reducir los impactos negativos de los choques económicos sobre las poblaciones más vulnerables. Además, se recalca la importancia de realizar este tipo de análisis a un nivel de desagregación temporal que permita capturar en forma adecuada las fluctuaciones que desaparecen al agregar los datos en forma temporal.

\section{REFERENCES}

Abbott, A., \& Jones, P. (2012a). Budget deficits and social protection: Cyclical government expenditure in the OECD. Economics Letters, 117(3), 909-911. https://doi.org/10.1016/j. econlet.2012.07.011

Abbott, A., \& Jones, P. (2012b). Intergovernmental transfers and procyclical public spending. Economics Letters, 115(3), 447-451. https://doi.org/10.1016/j.econlet.2011.12.104

Abbott, A., \& Jones, P. (2014). 'Leaning Against an Open Door': Ideology and the cyclicality of public expenditure. Journal of Policy Modeling, 36(6), 957-969. https://doi.org/10.1016/j. jpolmod.2014.09.003

Ahuja, D., \& Murthy, V. (2017). Social cyclicality in Asian countries. International Journal of Social Economics, 44(9), 1154-1165. https://doi.org/10.1108/IJSE-04-2016-0112

Alesina, A., Perotti, R., Giavazzi, F., \& Kollintzas, T. (1995). Fiscal Expansions and Adjustments in OECD Countries. Economic Policy, 10(21), 205. https://doi.org/10.2307/1344590

Aprobación del Protocolo de enmienda al Contrato suscrito entre el Gobierno de la República de Costa Rica y el instituto Interamericano de Cooperación para la Agricultura (IICA) sobre el Centro Agronómico Tropical de Investigación y Enseñanza (CATIE). (Ley Nº 8787, 2009). Costa Rica

Arreaza, A., Sorensen, B., \& Yosha, O. (1998). Consumption Smoothing through Fiscal Policy in OECD and EU Countries (No. w6372). Cambridge, MA: National Bureau of Economic Research. https://doi.org/10.3386/w6372

Arze del Granado, J., Gupta, S., \& Hajdenberg, A. (2013). Is Social Spending Procyclical? Evidence for Developing Countries. World Development, 42, 16-27. https://doi.org/10.1016/j. worlddev.2012.07.003 
FODESAF (2016). Programas por institución. Recuperado de http://fodesaf.go.cr/prog_soc_ selectivos/index.html

FODESAF (2018). Informe del registro de beneficiarios del 2016. Recuperado de http://fodesaf. go.cr/acerca_del_fodesaf/transparencia/estudios_iice/Informe $\% 20 \mathrm{de} \% 20$ registro $\% 20 \mathrm{de} \% 20$ beneficiarios\%202016\%20-\%2019\%20ABRIL.pdf

Forbes, K., \& Rigobon, R. (1999). No Contagion, Only Interdependence: Measuring Stock Market Co-movements (No. w7267). Cambridge, MA: National Bureau of Economic Research. https:// doi.org/10.3386/w7267

Furceri, D. (2010). Stabilization effects of social spending: Empirical evidence from a panel of OECD countries. The North American Journal of Economics and Finance, 21(1), 34-48. https://doi. org/10.1016/j.najef.2009.11.007

Lane, P. R. (2003). The cyclical behaviour of fiscal policy: evidence from the OECD. Journal of Public Economics, 87(12), 2661-2675. https://doi.org/10.1016/S0047-2727(02)00075-0

Ley de Desarrollo Social y Asignaciones Familiares (Ley $\mathrm{N}^{\circ}$ 5662, 2009). Costa Rica

Prasad, N., \& Gerecke, M. (2010). Social Security Spending in Times of Crisis. Global Social Policy: An Interdisciplinary Journal of Public Policy and Social Development, 10(2), 218-247. https:// doi.org/10.1177/1468018110366627

Ramírez, E. R. (1997). Costa Rica: Desempeño macroeconómico en 1996. Economía y Sociedad, $1(05), 111-118$.

Reforma Ley de Desarrollo Social y Asignaciones Familiares, $N^{\circ}$ 5662, Ley Pensión para Discapacitados con Dependientes $\mathrm{N}^{\circ} 7636$, Ley Creación del ICODER, N. . 7800, Ley Sistema Financiero Nacional para Vivienda $\mathrm{N}^{\circ} 7052$ y Ley Creación Fondo Nacional de Becas (Ley $\mathrm{N}^{\circ}$ 8783, 2009). Costa Rica

Sauma F., P., Sánchez Cantillo, M. V., Chacón, P., \& Sáenz, O. (2010). Implicaciones de la política macroeconómica, los choques externos y los sistemas de protección social en la pobreza, la desigualdad y la vulnerabilidad en América Latina y el Caribe: Costa Rica (Documentos de Proyectos No. 3). CEPAL. Recuperado de http://hdl.handle.net/11362/4175

Sørensen, B. E., Wu, L., \& Yosha, O. (2001). Output fluctuations and fiscal policy: U.S. state and local governments 1978-1994. European Economic Review, 45(7), 1271-1310. https://doi. org/10.1016/S0014-2921(00)00104-5

Stein, E., Talvi, E., \& Grisanti, A. (1998). Institutional Arrangements and Fiscal Performance: The Latin American Experience (No. w6358). Cambridge, MA: National Bureau of Economic Research. https://doi.org/10.3386/w6358 